\title{
Comparison of Ischemic Preconditioning and Intermittent and Continuous Inflow Occlusion in the Murine Liver
}

\author{
Hannes A. Rüdiger, MD, ${ }^{*}+$ Koo-J. Kang, MD, ${ }^{*}$ David Sindram, MSc, ${ }^{*}$ Hans-M. Riehle, MD, $\ddagger$ and \\ Pierre-A. Clavien, MD, PhD, FACS*†
}

From the *Department of Surgery, Duke University Medical Center, Durham, North Carolina, and the Departments of +Visceral and Transplant Surgery and ¥Pathology, University Hospital Zürich, Switzerland

\section{Objective}

To compare protection of the liver by ischemic preconditioning and intermittent inflow occlusion in a mouse model of prolonged periods of ischemia.

\section{Summary Background Data}

Preconditioning (short ischemic stress prior to a prolonged period of ischemia) and intermittent inflow occlusion protect the liver against reperfusion injury. This is the first study comparing these two modalities with continuous inflow occlusion (control).

\section{Methods}

Mice were subjected to 75 or 120 minutes of $70 \%$ hepatic ischemia and 3 hours of reperfusion. Each ischemic period was evaluated using three different protocols: continuous ischemia (control), preconditioning (10 minutes ischemia and 15 minutes reperfusion) prior to the prolonged ischemic insult, and intermittent clamping (cycles of 15 minutes ischemia and 5 minutes reperfusion). Organ injury was evaluated using serum levels of aspartate aminotransferase (AST), hematoxylin and eosin staining, and specific markers of apoptosis (cytochrome $\mathrm{C}$ release, caspase 3 activity, and TUNEL staining). Animal survival was determined using a model of total hepatic ischemia.

\section{Results}

Intermittent inflow occlusion and ischemic preconditioning were both protective against ischemic insults of 75 and 120 minutes compared with controls (continuous ischemia only). Protection against 75 minutes of ischemia was comparable in the intermittent clamping and the ischemic preconditioning group, whereas intermittent clamping was superior at 120 minutes of ischemia. One hundred percent animal survival was observed after 75 minutes of total hepatic ischemia using both protective protocols, whereas all animals subjected to continuous ischemia died after surgery. After 120 minutes of ischemia, intermittent inflow occlusion was associated with better animal survival (71\%) compared with preconditioning (14\%).

\section{Conclusions}

Preconditioning and intermittent clamping are both protective against prolonged periods of ischemia. In the clinical setting, preconditioning is superior for ischemic periods of up to 75 minutes because it is not associated with blood loss during transection of the liver. However, for prolonged ischemic insults exceeding 75 minutes, intermittent clamping is superior to preconditioning.
Major liver resections are associated with a significant risk of bleeding. Strategies to prevent blood loss during transection of the hepatic parenchyma include vascular oc-

Drs. Rüdiger and Kang contributed equally to this work.

Supported by grants from the National Institutes of Health (DK54048 to P.A.C.) and the Swiss National Science Foundation (SNF3200061411). Dr. Rüdiger is supported by grants from the Swiss National Science Foundation (grant 81ZH-059608), the Roche Research Foundation, Switzerland, and the Olga Nayer Fisch Foundation, Switzerland. clusion either by inflow (Pringle maneuver) or by a combination of inflow and outflow (total vascular exclusion) occlusion. $^{1-6}$ Because the liver poorly tolerates prolonged periods of ischemia with irreversible damage occurring

Correspondence: Pierre-A. Clavien, MD, PhD, FACS, Department of Visceral and Transplant Surgery, University Hospital Zürich, Rämistrasse 100, 8091 Zürich, Switzerland.

E-mail: clavien@chir.unizh.ch

Accepted for publication August 30, 2001. 
about 1 hour after interruption of the blood supply, various protective strategies have been developed. Intermittent inflow occlusion (intermittent Pringle maneuver) ${ }^{7,8}$ and ischemic preconditioning ${ }^{9}$ extend safe periods of ischemia during hepatic surgery.

Intermittent inflow occlusion, first reported by Makuuchi et al in $1987,{ }^{8}$ has gained wide popularity, particularly in Asia. Various protocols have been recommended using periods of inflow occlusion ranging between 10 and 30 minutes followed by periods of 5 to 15 minutes of reperfusion. ${ }^{8,10-12} \mathrm{~A}$ number of experimental ${ }^{10,11,13}$ and clinical ${ }^{7,8,14-16}$ studies have shown better tolerance of the liver to intermittent clamping of the portal triad compared with continuous inflow occlusion. For example, van Wagensveld et $\mathrm{al}^{13}$ showed in a pig model that intermittent clamping dramatically reduced serum levels of aspartate aminotransferase (AST) with improved uptake of hyaluronic acid, suggesting better sinusoidal endothelial cell function, protection of hepatocytes, and improved microcirculation. ${ }^{13}$ A randomized clinical study in liver resection patients by Belghiti et $\mathrm{al}^{7}$ showed that intermittent clamping using multiple cycles of 15 minutes of ischemia and 5 minutes of reperfusion is associated with decreased injury compared with similar periods of continuous inflow occlusion. In Japan, ischemic times up to 320 minutes using intermittent clamping were safely used in patients undergoing major liver resection. ${ }^{17}$ However, drawbacks associated with intermittent occlusion include blood loss from the transected surface of the liver during the successive periods of reperfusion ${ }^{7}$ and increased duration of surgery. In addition, although the protective effects of intermittent clamping have been repeatedly shown, our insight into the mechanisms of protection remains speculative.

Ischemic preconditioning has been applied only recently to hepatic surgery. ${ }^{9}$ The concept of ischemic preconditioning is based on the biologic principle that tissue primed by various types of sublethal stress develops tolerance to subsequent lethal injury. ${ }^{18}$ In 1986 , Murry et a ${ }^{19}$ identified in the heart the protective potential of a short period of ischemia (ischemic preconditioning) in preventing injury from subsequent prolonged periods of ischemia. The protective effects of ischemic preconditioning were subsequently described in various tissues, including the liver. ${ }^{20,21}$ We recently demonstrated in a mouse model that ischemic preconditioning protects against lethal ischemic stress through downregulation of the apoptotic pathway. ${ }^{22}$ Various mediators have been implicated in the protective mechanisms of ischemic preconditioning (e.g., nitric oxide, ${ }^{20}$ adenosine, ${ }^{23}$ and others ${ }^{24}$ ). We recently provided evidence that a preconditioning period of 10 minutes followed by 10 minutes of reperfusion confers protection against prolonged ischemic insults in patients undergoing liver resections. ${ }^{9}$ One advantage of ischemic preconditioning over intermittent clamping is the absence of blood loss associated with this technique. ${ }^{9}$

Although strong evidence now exists regarding the protective effects of intermittent inflow occlusion and ischemic preconditioning, no comparative study is currently avail- able. Therefore, we evaluated the effects and underlying mechanisms of intermittent clamping and ischemic preconditioning in a model of prolonged ischemic injury in the murine liver. Animals undergoing continuous hepatic inflow occlusion served as controls.

\section{METHODS}

\section{Study Design}

The experimental groups included intermittent clamping and ischemic preconditioning prior to a continuous ischemic insult. Continuous occlusion without protection served as controls. Two different periods of continuous occlusion were tested. First, an interval of 75 minutes of hepatic ischemia was chosen because it represents the minimal insult associated with consistent animal death. ${ }^{22}$ Second, an ischemic interval of 120 minutes was chosen, which is beyond the limits for animal survival after ischemic preconditioning in our model. ${ }^{22}$

\section{Protocols of Organ Protection}

We have previously tested various protocols of ischemic preconditioning. ${ }^{22}$ In this study, we chose the most effective protocol of ischemic preconditioning, which consisted of 10 minutes of ischemia followed by 15 minutes of reperfusion followed by the prolonged ischemic insult. ${ }^{22}$ Intermittent clamping consisted of five or eight cycles of 15 minutes of ischemia followed by 5 minutes of reperfusion. This is currently the most popular clinical protocol used in experienced centers. ${ }^{7}$

\section{Animals}

Male wild-type mice (C57BL6, Jackson Lab, Bar Harbor, $\mathrm{ME})$ were used in all experiments. Animals were fed a laboratory diet with water and food ad libitum until use and were kept under constant environmental conditions with 12-hour light/dark cycles. All procedures were performed in accordance with Duke Institutional Animal Care Committee guidelines.

\section{Partial Hepatic Ischemia}

A nonlethal model of segmental (70\%) hepatic ischemia was used as previously described. ${ }^{25}$ Surgery was performed under isoflurane anesthesia (Halocarbon Laboratories, River Edge, NJ). Briefly, the abdomen was entered through a midline incision and the liver was freed from its ligaments. The portal triad was dissected and a microvascular clamp (Aesculap, San Francisco, CA) was placed, conferring ischemia to the median and the left lobes. Mesenteric congestion was avoided by allowing intestinal blood flow through the right and caudate lobes. During ischemic periods, the abdomen was closed by running suture with 4-0 chromic gut 
(Ethicon, Somerville, NJ). The animal was kept under anesthesia on reperfusion. Reperfusion was initiated by removing the clamp. The animal was allowed to recover from anesthesia during the 3 hours of reperfusion.

\section{Total Hepatic Ischemia}

Animal survival was evaluated using a modification of our previously described technique of total hepatic ischemia. ${ }^{26}$ Briefly, after the ischemic interval the abdomen was reopened and the microclamp was removed. The right and caudate lobes were then resected, leaving only ischemic tissue in place. The abdomen was closed and the animals recovered from anesthesia and were closely monitored for 7 days.

\section{Serum Enzyme Analysis}

Serum levels of AST were used as a general marker of hepatocyte injury. Blood samples were obtained after 3 hours of reperfusion from the inferior vena cava. Blood cells were pelleted by immediate centrifugation at $8,000 \mathrm{~g}$ for 10 minutes. Enzyme levels were measured using the serum multiple biochemical analyzer (Ektachem DTSCII, Johnson \& Johnson, Rochester, NY).

\section{TUNEL Staining}

Apoptosis leads to specific DNA strand breaks that can be labeled with modified nucleotides in an enzymatic reaction involving terminal deoxynucleotidyl transferase (TUNEL). Livers underwent in vivo fixation by perfusion with $4 \%$ paraformaldehyde (Sigma, St. Louis, MO) in phosphatebuffered saline through the portal vein. Frozen sections (5 $\mu \mathrm{m})$ of the fixed tissue were prepared and stained by the TUNEL method using a commercial kit (Boehringer Mannheim Co., Indianapolis, IN) according to the manufacturer's recommendations. Morphometric analysis of the fluorescent cells was performed under high-power magnification $(400 \times)$ in a blinded fashion.

\section{Caspase Activity}

Caspase- 3 activity was determined by measuring proteolytic cleavage of the specific substrate $n$-acetyl-Asp-GluVal-Asp-7-amino-4-trifluoromethyl coumarin (Ac-DEVDAFC; Biomol, Plymouth Meeting, PA) in the presence or absence of the specific inhibitor $n$-acetyl-Asp-Glu-Val-AspCHO (Ac-DEVD-CHO; Biomol). Liver tissue was quickly excised and sonicated in assay buffer $(1 \mathrm{mmol} / \mathrm{L}$ ethylenediaminetetraacetic acid, $145 \mathrm{mmol} / \mathrm{L} \mathrm{NaCl}, 100 \mathrm{mmol} / \mathrm{L}$ Tris, $0.1 \mathrm{mmol} / \mathrm{L}$ DTT, $0.1 \%$ CHAPS, $10 \%$ glycerol [all from Sigma]). Protein content was determined using the Bradford protein assay (Biorad, Hercules, CA). The samples were incubated at room temperature with Ac-DEVDAFC substrate in the presence or absence of the inhibitor
Ac-DEVD-CHO. AFC release is expressed as arbitrary fluorescence units per milligram of liver tissue after subtracting the reading in the inhibited sample from the noninhibited sample.

\section{Cytochrome C Release}

The cells were homogenized in $10 \mathrm{vol} / \mathrm{wt}$ assay buffer (20 $\mathrm{mmol} / \mathrm{L}$ HEPES-KOH [pH 7.4], $10 \mathrm{mmol} / \mathrm{L} \mathrm{KCl}, 1.5 \mathrm{mmol} / \mathrm{L}$ $\mathrm{MgCl}_{2}, 1 \mathrm{mmol} / \mathrm{L}$ EDTA, $1 \mathrm{mmol} / \mathrm{L}$ EGTA, $1 \mathrm{mmol} / \mathrm{L}$ DTT, $0.1 \mathrm{mmol} / \mathrm{L}$ PMSF, $250 \mathrm{mmol} / \mathrm{L}$ sucrose [all from Sigma]) using a Dounce grinder (Kontess Glass Co., Vineland, NJ). Unbroken cells and nuclei were pelleted at $400 \mathrm{~g}$ for 5 minutes. The supernatants were further centrifuged at $12,000 \mathrm{~g}$ for 10 minutes at $4^{\circ} \mathrm{C}$ to pellet the mitochondria. The supernatant was boiled with an equal volume of $2 \times$ SDS sample buffer ${ }^{27}$ for 90 seconds and subjected to $4 \%$ to $20 \%$ SDS-PAGE (Novex, San Diego, CA). The proteins were transferred onto a nitrocellulose filter. Blots were probed with a rabbit polyclonal anticytochrome C antibody (Santa Cruz Biotechnology, Santa Cruz, CA) followed by a secondary antibody conjugated to horseradish peroxidase and detected with enhanced chemiluminescence detection reagents (Amersham, Piscataway, NJ).

\section{Statistical Analysis}

Results are expressed as means \pm standard error. Data were analyzed using SPSS software version 9.0.0 (SPSS Inc., Chicago, IL). Differences between the groups were evaluated using analysis of variance, and then pairwise comparisons were performed with the Tukey post hoc procedure. Animal survival was compared using the Fisher exact test. $P \leq .05$ was considered statistically significant.

\section{RESULTS}

\section{Effects of Ischemic Preconditioning and Intermittent Inflow Occlusion on Hepatocellular Injury After Prolonged Ischemia}

The effects of ischemic preconditioning and intermittent clamping were evaluated in a mouse model of partial hepatic ischemia. Serum transaminase levels, an established marker of hepatocyte injury, ${ }^{28}$ and liver tissue were analyzed 3 hours after reperfusion in animals subjected either to 75 or 120 minutes of ischemia. Each group included five animals.

Levels of AST were significantly lower in animals subjected to ischemic preconditioning and intermittent clamping after 75 minutes of ischemia compared with mice treated with 75 minutes of continuous clamping only $(P<$ .001 , analysis of variance) (Fig. 1). AST levels were comparable in the ischemic preconditioning and intermittent inflow occlusion groups ( $P=.4$, Tukey). After 120 minutes of ischemia, AST levels were lower in the intermittent 


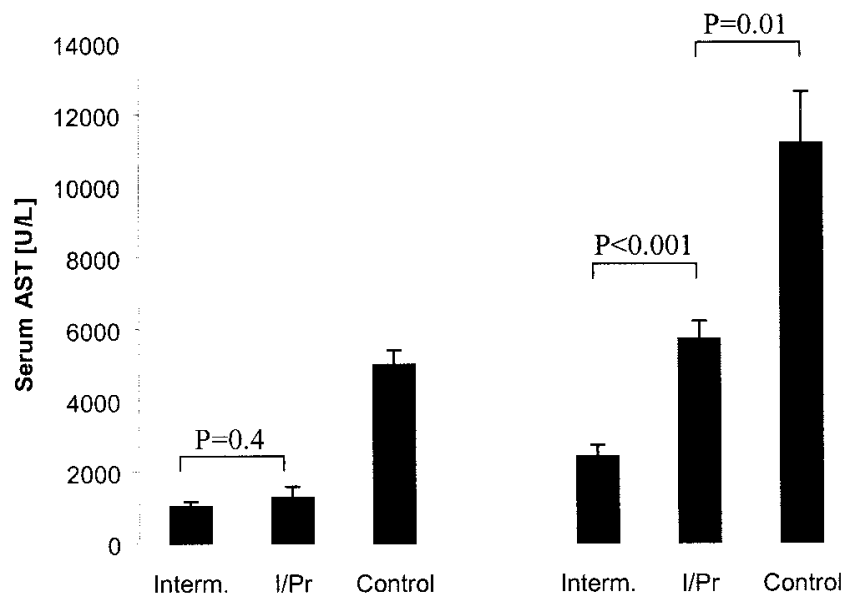

$75 \mathrm{~min}$ ischemia

$120 \mathrm{~min}$ ischemia

Figure 1. Serum levels of aspartate transaminase (AST) served as markers of hepatocyte injury. AST levels were measured after 75 or 120 minutes of ischemia and 3 hours of reperfusion. Similar protection was observed after 75 minutes of ischemia in the intermittent clamping group (Interm.) and the ischemic preconditioning group (I/Pr) $(P=.4$, Tukey). After 120 minutes of ischemia, intermittent clamping was superior to both other groups. Continuous ischemia (Control) was associated with the highest AST levels after both ischemic periods $(P<.001$, analysis of variance) (mean \pm standard error, $n=5$ per group).

occlusion group compared with both other groups $(P<$ .001 , analysis of variance). However, levels in the ischemic preconditioning animals remained significantly lower than in the controls $(P=.01$, Tukey $)$.

Morphologic changes in the parenchyma were determined using liver sections stained with hematoxylin and eosin after 3 hours of reperfusion. The parenchymal appearance was normal after ischemic preconditioning and intermittent clamping with an ischemic insult of 75 minutes of ischemia. In contrast, after 75 minutes of continuous ischemia, we observed large confluent areas of tissue destruction with blood congestion in the sinusoids, cellular lysis, and cytoplasmic vacuolization with minimal inflammatory infiltrations (Fig. 2). A similar pattern of parenchymal destruction was observed in the ischemic preconditioning group after 120 minutes of continuous inflow occlusion. In contrast, the parenchyma was well preserved in the intermittent clamping group after 120 minutes of ischemia.

\section{Effects of Ischemic Preconditioning and Intermittent Inflow Occlusion on Apoptotic Cell Death}

Apoptosis of hepatocytes is a central feature of cell death after reperfusion of the warm ischemic liver. ${ }^{22,29,30} \mathrm{We}$ recently found that the protective effects of ischemic preconditioning involved dramatic protection against apoptosis. $^{22}$ Liver samples were evaluated 3 hours after reperfusion in each group because the degree of apoptosis is maximal at this time point. $^{22}$

We used the TUNEL assay to quantify the number of apoptotic hepatocytes with characteristic DNA fragmentation. When compared with continuous occlusion, both isch-

\section{Intermittent Clamping}
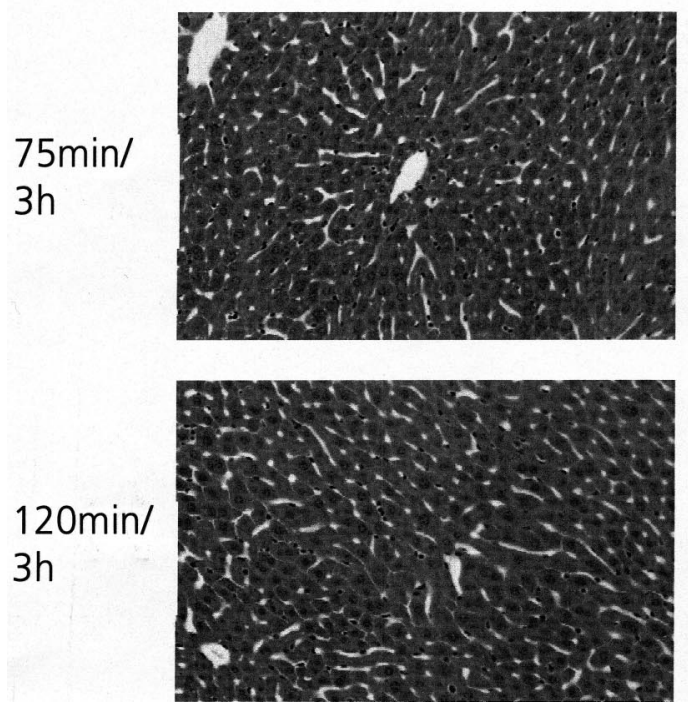

Preconditioning
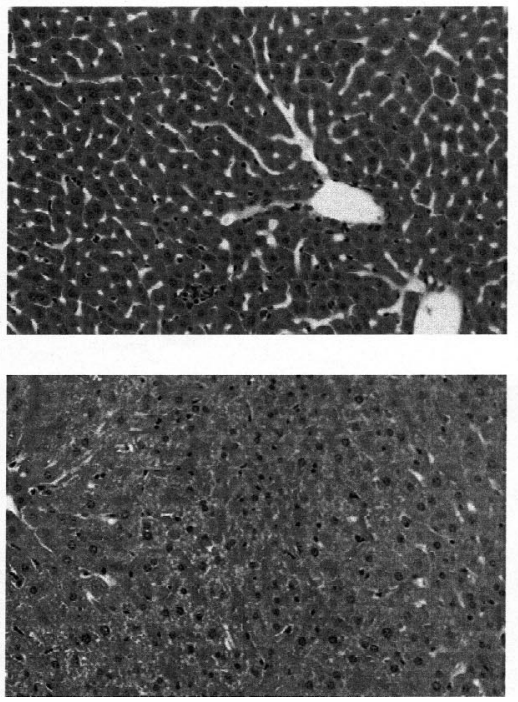

Control
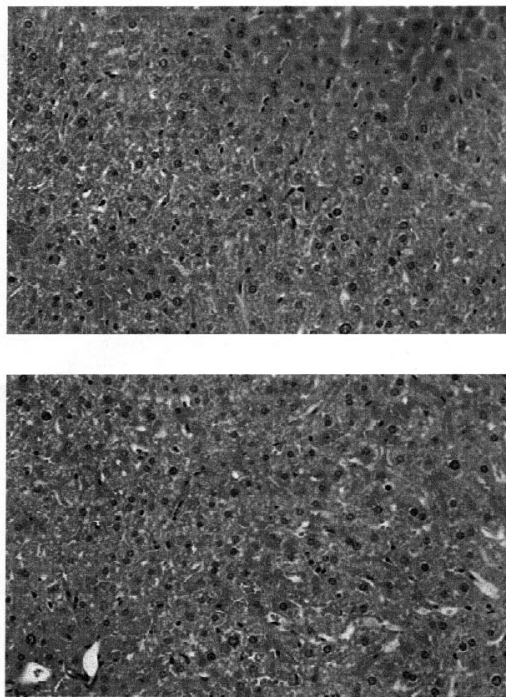

Figure 2. Tissue sections stained with hematoxylin and eosin. Representative histologic appearance of livers subjected to either 75 or 120 minutes of ischemia and 3 hours of reperfusion. Normal parenchyma was observed after ischemic insults of 75 and 120 minutes using intermittent clamping and after 75 minutes with preconditioning. Severe parenchymal alterations were observed in the control groups (75 and 120 minutes of continuous ischemia only) and with ischemic preconditioning in livers subjected to 120 minutes of ischemia. 


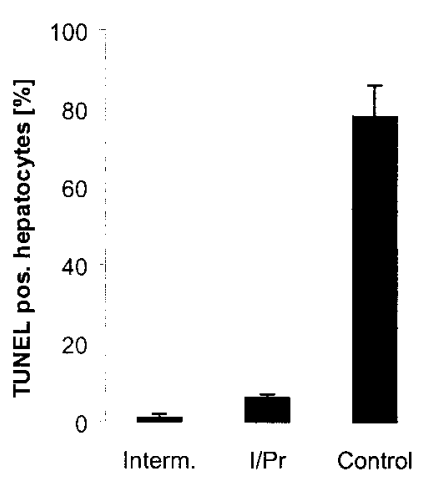

75 min ischemia

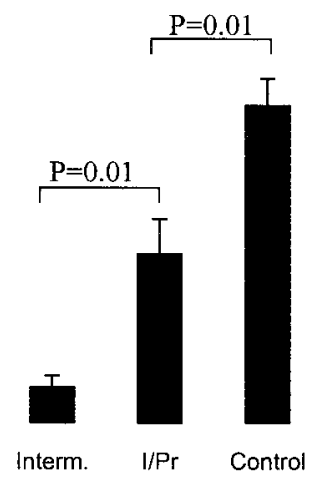

$120 \mathrm{~min}$ ischemia

Figure 3. TUNEL staining was used to quantify the degree of apoptosis in liver tissue after either 75 or 120 minutes of ischemia and 3 hours of reperfusion. Intermittent clamping (Interm.) and ischemic preconditioning $(1 / \mathrm{Pr})$ were both associated with a minimal number of apoptotic hepatocytes after 75 minutes of ischemia. Ischemic preconditioning did not completely prevent apoptosis after 120 minutes of ischemia but was still significantly lower than the controls (continuous ischemia only) $(P=.001$, analysis of variance; $\mathrm{n}=5$ per group; mean \pm standard error).

emic preconditioning and intermittent clamping were associated with complete prevention of apoptosis after 75 minutes of ischemia (Fig. 3). After 120 minutes of ischemia, the protective effects of ischemic preconditioning against apoptosis were partially lost, whereas the degree of apoptosis remained low in the intermittent clamping group.

\section{Effects of Ischemic Preconditioning and Intermittent Inflow Occlusion on Cellular Mediators of Apoptosis}

To evaluate further the mechanisms leading to apoptotic cell death, we measured two established mediators of apoptosis in each group, the release of cytochrome $\mathrm{C}$ from the mitochondria into the cytoplasm, and cytoplasmic caspase-3 activity. Both intermittent clamping and ischemic preconditioning significantly prevented the release of cytochrome $\mathrm{C}$ after 75 and 120 minutes of ischemia compared with the respective controls (continuous clamping) (Fig. 4). The activity of caspase-3, which is located further downstream of the apoptotic cascade, was also significantly decreased

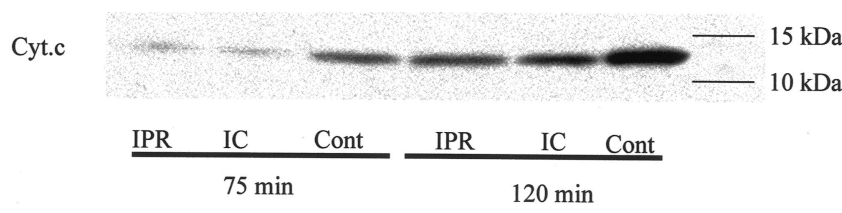

Figure 4. Cytoplasmic cytochrome C (Cyt.c). Hepatic protection by ischemic preconditioning (IPR) and intermittent clamping (IC) prevented cytochrome $\mathrm{C}$ release from the mitochondria into the cytoplasm after 75 and 120 minutes of ischemia and 3 hours of reperfusion compared to the controls (Cont; continuous ischemia only) as assessed by Western blot analysis.

50

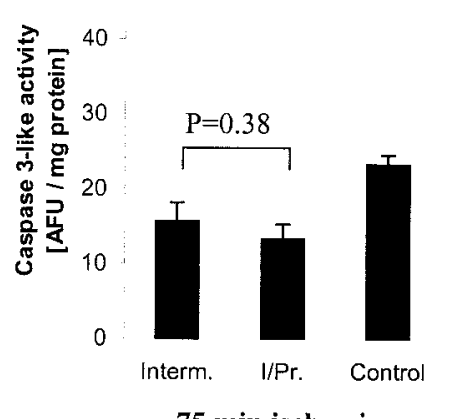

75 min ischemia

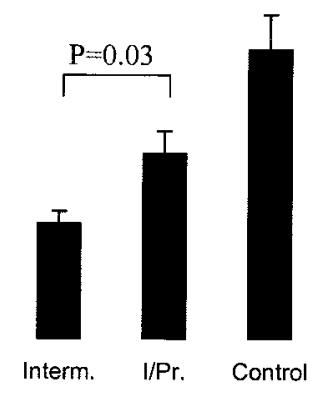

$120 \mathrm{~min}$ ischemia
Figure 5. Caspase-3-like activity was evaluated in tissue after 3 hours of reperfusion using a fluorometric assay. After 75 minutes of ischemia, no difference was observed between intermittent clamping (Interm.) and ischemic preconditioning $(1 / \mathrm{Pr})(P=.38$, Tukey). After an ischemic insult of 120 minutes, caspase-3-like activity was significantly lower in the intermittent clamping group compared with the ischemic preconditioning group ( $P=.03$, Tukey). The control groups (continuous ischemia only) were associated with the highest caspase-3 activities after both ischemic periods $(P=.01$ after 75 minutes and $P=.001$ after 120 minutes, analysis of variance). AFU, arbitrary fluorescence units; $n=5$ per group; mean \pm standard error.

after intermittent clamping and ischemic preconditioning after both ischemic intervals $(P=.01$ after 75 minutes and $P=.001$ after 120 minutes, analysis of variance, $\mathrm{n}=5$ ). However, caspase-3 activity was significantly higher after 120 minutes of ischemia in the ischemic preconditioning group compared with values in the intermittent clamping group ( $P=.03$, Tukey) (Fig. 5).

\section{Effects of the Various Modalities of Inflow Occlusion on Animal Survival}

To confirm the clinical relevance of the biochemical and histologic findings, we evaluated animal survival using our model of total hepatic ischemia. After 75 minutes of ischemia, consistent animal survival (100\% [7/7]) was observed with both ischemic preconditioning and intermittent clamping. In contrast, all animals undergoing continuous ischemia died within 3 days of surgery (Table 1$)(P<.001$, Fisher exact test, $\mathrm{n}=7$ ). After 120 minutes of ischemia, the intermittent clamping protocol was still associated with

\begin{tabular}{|c|c|c|}
\hline \multicolumn{3}{|c|}{ Table 1. ANIMAL SURVIVAL* } \\
\hline Ischemia time & $75 \mathrm{~min}$ & $120 \mathrm{~min}$ \\
\hline Continuous inflow occlusion (control) & ०\% (0/7) & $0 \%(0 / 7)$ \\
\hline $\begin{array}{l}\text { Ischemic preconditioning before the } \\
\text { prolonged ischemic insult }\end{array}$ & $100 \%(7 / 7) \dagger$ & $14.3 \%(1 / 7)$ \\
\hline Intermittent clamping & $100 \%(7 / 7) \dagger$ & $71.4 \%(5 / 7) \dagger$ \\
\hline \multicolumn{3}{|c|}{$\begin{array}{l}{ }^{*} \text { Animal survival was evaluated using our model of total hepatic ischemia. }{ }^{26} \\
\mathrm{n}=7 ; \dagger P \leq .05 \text { vs. controls, Fisher exact test. }\end{array}$} \\
\hline
\end{tabular}


$71 \%(5 / 7)$ animal survival, whereas it was poor in the preconditioning group (14\% [1/7]) and after continuous occlusion only $(0 \%[0 / 7])$.

\section{DISCUSSION}

Intermittent inflow occlusion ${ }^{7,8}$ and ischemic preconditioning 9,20,22 protect the liver against long periods of ischemia. In this study, we compared these two strategies with animals subjected to periods of continuous inflow occlusion only. Based on various parameters of tissue injury and animal survival, we found that ischemic preconditioning is comparable to intermittent clamping for ischemic times of up to 75 minutes. However, for longer periods of ischemia (120 minutes), intermittent clamping appeared superior to ischemic preconditioning.

Most experienced centers in hepatobiliary surgery use periods of inflow occlusion of less than 75 minutes for most types of hepatic resection. ${ }^{5-7}$ In these cases, ischemic preconditioning seems to be the preferred modality of protection. Although ischemic preconditioning and intermittent clamping offer the same degree of parenchymal protection, ischemic preconditioning is not associated with the increased risk of blood loss inherent to techniques of intermittent clamping ${ }^{7,31}$ and does not extend the duration of surgery. We recently demonstrated that ischemic preconditioning could be easily performed during preparation of the liver for resection, and thus without having a negative impact on the duration of surgery. ${ }^{9}$

A growing body of evidence suggests that protective strategies are advised even for short periods of ischemia. ${ }^{9}$ In a recent clinical study in liver resection patients, we found that ischemic preconditioning is protective against $30 \mathrm{~min}$ utes of ischemia, with reduction of serum transaminase levels more than twofold compared with a group of patients subjected to continuous ischemia only. ${ }^{9}$ From the data presented here, ischemic preconditioning appears to be the best-known protective modality. For periods of hepatic ischemia beyond 75 minutes, intermittent clamping was superior to ischemic preconditioning in our model. However, only a few protective modalities have been tested so far for the uncommon situation of very long periods of ischemia. More efficient protocols might be developed, for example with strategies combining the two protocols. Because ischemic preconditioning is highly effective against a period of up to 75 minutes, we could imagine beginning with an ischemic preconditioning protocol and then switching to intermittent clamping when the ischemic time exceeds 75 minutes. Of note, the tolerance of the murine liver against ischemia appears to be comparable to that of the human liver. ${ }^{9,22}$ Therefore, it could be conceivable to design a safe human study in patients undergoing liver resection to identify the best strategy. The aim of such studies would be to find a balance between parenchymal protection and blood loss with minimal impact on the duration of surgery.

Our recent studies in the ischemic rodent ${ }^{22}$ and human ${ }^{9}$ liver have pointed to apoptosis as a central feature of hepatocyte injury. Ischemic preconditioning was subsequently found to confer protection through downregulation of caspase activities and apoptosis. ${ }^{22}$ Therefore, a focus of this study was on the evaluation of the apoptotic pathway in the model of intermittent clamping. All specific markers for apoptosis (i.e., TUNEL staining, cytochrome $\mathrm{C}$ release, and caspase-3 activities) were decreased after intermittent clamping or ischemic preconditioning prior to the prolonged ischemic insult. These findings correlated with the decrease in serum AST levels and improved animal survival. Taken together, downregulation of apoptotic cell death appears to be a central mechanism of protection, rather than only an epiphenomenon. ${ }^{24}$

Staining with hematoxylin and eosin was used to evaluate morphologic changes in the postischemic liver tissue. In tissue subjected to continuous inflow occlusion (with either 75 or 120 minutes of ischemia) and in the preconditioning group with 120 minutes of ischemia, we observed a pattern of tissue destruction that could also be a result of necrotic changes. However, all the specific markers studied pointed to apoptosis as the major mechanism of tissue injury in our model. The involvement of mediators of apoptosis has also been suggested by others. ${ }^{32-34}$ For example, Cursio et $\mathrm{al}^{33}$ found that inhibition of caspases protects against reperfusion injury after normothermic ischemia. In addition, we $\mathrm{e}^{35}$ and others ${ }^{34}$ showed that overexpression of the antiapoptotic protein $\mathrm{Bcl}-2$ is highly protective against reperfusion injury in the ischemic liver. It is therefore possible that the morphologic changes observed in the stained slides might be the results of rapid and massive development of apoptosis involving large areas of the ischemic lobes. The intense hypoxic stress from the ischemic insult may lead to this confluent tissue alteration, which contrasts with the disseminated destruction of single cells observed in other models of in vivo hepatocyte apoptosis (e.g., chronic hepatitis $\mathrm{C}^{36}$ or hepatic allograft rejection ${ }^{37}$ ).

Although we are beginning to understand the basic pathways of ischemic preconditioning,,$^{2,23,38}$ our insight into the mechanisms of intermittent clamping was limited. This study provides the first evidence that intermittent clamping, as ischemic preconditioning, induces inhibition of apoptosis with prevention of cytochrome $\mathrm{C}$ release and caspase- 3 activation. However, the triggering mechanisms of inhibition of the apoptotic pathway remain elusive. Intermittent clamping seems to initiate additional factors conferring persistent protection. The successive periods of reperfusion may provide the cells with a sufficient energy reserve to initiate and maintain synthesis or activation of protective factors, such as heat shock proteins or transcription factors, including heme oxygenase- $1,{ }^{18,39} \mathrm{AP}-1,{ }^{40}$ or $\mathrm{NF} \kappa \mathrm{B} .{ }^{41} \mathrm{An}-$ other possible mechanism might involve repeated flushing of the liver, with resulting washout of harmful factors from the vascular bed (e.g., activated blood cells, ${ }^{42,43}$ oxygen free radicals, ${ }^{43}$ or proinflammatory cytokines $\left.{ }^{44}\right)$. Accumulation 
of these factors might explain the failure of ischemic preconditioning to protect against long ischemic insults.

Patients with chronic liver diseases (cirrhosis or steatosis) remain a major challenge in hepatic surgery. It has been suggested that steatotic ${ }^{45,46}$ and cirrhotic ${ }^{45,47,48}$ livers are particularly susceptible to ischemic insults. Therefore, the need for organ protection against ischemic injury is of particular importance in this population. However, neither intermittent clamping nor ischemic preconditioning has been thoroughly tested in the setting of chronic liver disease, but limited data suggest efficient organ protection with both modalities. $\mathrm{We}^{9}$ and Belghiti et $\mathrm{al}^{7}$ found significant protection by ischemic preconditioning and intermittent inflow occlusion, respectively, in patients with hepatic steatosis as determined by transaminase levels. Efficient organ protection might open new avenues in the treatment of this large patient population.

In conclusion, this study indicates that organ protection through ischemic preconditioning is comparable to intermittent clamping for ischemic periods of up to 75 minutes. Ischemic preconditioning is probably superior in the clinical setting because it is not associated with blood loss during the multiple cycles of unclamping. Tissue protection through intermittent clamping is superior for ischemic periods exceeding 75 minutes. These results should now lead to clinical trials.

\section{References}

1. Pringle J. Notes on the arrest of hepatic hemorrhage due to trauma. Ann Surg 1908; 48:541-549.

2. Belghiti J, Noun R, Zante E, et al. Portal triad clamping or hepatic vascular exclusion for major liver resection. A controlled study. Ann Surg 1996; 224:155-161.

3. Man K, Fan ST, Ng IO, et al. Prospective evaluation of Pringle maneuver in hepatectomy for liver tumors by a randomized study. Ann Surg 1997; 226:704-713.

4. Terblanche J, Krige JE, Bornman PC. Simplified hepatic resection with the use of prolonged vascular inflow occlusion. Arch Surg 1991; 126:298-301.

5. Cunningham JD, Fong Y, Shriver C, et al. One hundred consecutive hepatic resections. Blood loss, transfusion, and operative technique. Arch Surg 1994; 129:1050-1056.

6. Bismuth H, Castaing D, Garden OJ. Major hepatic resection under total vascular exclusion. Ann Surg 1989; 210:13-19.

7. Belghiti J, Noun R, Malafosse R, et al. Continuous versus intermittent portal triad clamping for liver resection: a controlled study. Ann Surg 1999; 229:369-375.

8. Makuuchi M, Mori T, Gunven P, et al. Safety of hemihepatic vascular occlusion during resection of the liver. Surg Gynecol Obstet 1987; 164:155-158.

9. Clavien PA, Yadav S, Sindram D, et al. Protective effects of ischemic preconditioning for liver resection performed under inflow occlusion in humans. Ann Surg 2000; 232:155-162.

10. Hardy KJ, Tancheroen S, Shulkes A. Comparison of continuous versus intermittent ischaemia-reperfusion during liver resection in an experimental model. Br J Surg 1995; 82:833-836.

11. Isozaki H, Adam R, Gigou M, et al. Experimental study of the protective effect of intermittent hepatic pedicle clamping in the rat. Br J Surg 1992; 79:310-313.
12. Horiuchi T, Muraoka R, Tabo T, et al. Optimal cycles of hepatic ischemia and reperfusion for intermittent pedicle clamping during liver surgery. Arch Surg 1995; 130:754-758.

13. van Wagensveld BA, van Gulik TM, Gelderblom HC, et al. Prolonged continuous or intermittent vascular inflow occlusion during hemihepatectomy in pigs. Ann Surg 1999; 229:376-384.

14. Man K, Fan ST, Ng IO, et al. Tolerance of the liver to intermittent Pringle maneuver in hepatectomy for liver tumors. Arch Surg 1999; 134:533-539.

15. Elias D, Desruennes E, Lasser P. Prolonged intermittent clamping of the portal triad during hepatectomy. Br J Surg 1991; 78:42-44.

16. Elias D, Lasser P, Debaene B, et al. Intermittent vascular exclusion of the liver (without vena cava clamping) during major hepatectomy. Br J Surg 1995; 82:1535-1539.

17. Sakamoto Y, Makuuchi M, Takayama T, et al. Pringle's maneuver lasting $322 \mathrm{~min}$. Hepato-Gastroenterology 1999; 46:457-458.

18. Rudiger HA, Clavien PA. A cytotoxic drug against reperfusion injury? Hepatology 2000; 31:533-535.

19. Murry CE, Jennings RB, Reimer KA. Preconditioning with ischemia: a delay of lethal cell injury in ischemic myocardium. Circulation 1986; 74:1124-1136.

20. Peralta C, Closa D, Hotter G, et al. Liver ischemic preconditioning is mediated by the inhibitory action of nitric oxide on endothelin. Biochem Biophys Res Commun 1996; 229:264-270.

21. Hardy K, McClure D, Subwongchareon S. Ischaemic preconditioning of the liver: a preliminary study. Aust NZ J Surg 1996; 66:707-710.

22. Yadav SS, Sindram D, Perry DK, et al. Ischemic preconditioning protects the mouse liver by inhibition of apoptosis through a caspasedependent pathway. Hepatology 1999; 30:1223-1231.

23. Peralta C, Hotter G, Closa D, et al. Protective effect of preconditioning on the injury associated to hepatic ischemia-reperfusion in the rat: role of nitric oxide and adenosine. Hepatology 1997; 25:934-937.

24. Bulkley G. Preconditioning for protection form ischemic injury: Discriminating cause from effect from epiphenomenon. Ann Surg 2000; 232:163-165.

25. Camargo CA Jr, Madden JF, Gao W, et al. Interleukin-6 protects liver against warm ischemia/reperfusion injury and promotes hepatocyte proliferation in the rodent. Hepatology 1997; 26:1513-1520.

26. Yadav SS, Gao W, Harland RC, et al. A new and simple technique of total hepatic ischemia in the mouse. Transplantation 1998; 65:14331436.

27. Laemmli UK. Cleavage of structural proteins during the assembly of the head of bacteriophage T4. Nature 1970; 227:680-685.

28. Iu S, Harvey P, Makowka L, et al. Markers of allograft viability in the rat. Relationship between transplantation viability and liver function in the isolated perfused rat liver. Transplantation 1987; 45:562-569.

29. Kohli V, Madden JF, Bentley RC, et al. Calpain mediates ischemic injury of the liver through modulation of apoptosis and necrosis. Gastroenterology 1999; 116:168-178.

30. Kohli V, Selzner M, Madden J, et al. Endothelial cell and hepatocyte deaths occur by apoptosis after ischemia-reperfusion injury in the rat liver. Transplantation 1999; 67:1099-1105.

31. Nagao T, Inoue S, Goto S, et al. Hepatic resection for hepatocellular carcinoma. Clinical features and long-term prognosis. Ann Surg 1987; 205:33-40.

32. Natori S, Selzner M, Valentino K, et al. Apoptosis of sinusoidal endothelial cells occurs during liver preservation injury by a caspasedependent mechanism. Transplantation 1999; 68:89-96.

33. Cursio R, Guggenheim J, Ricci J, et al. A caspase inhibitor fully protects rats against lethal normothermic liver ischemia by inhibition of liver apoptosis. FASEB J 1999; 13:253-256.

34. Bilbao G, Contreras J, Eckhoff D, et al. Reduction of ischemiareperfusion injury of the liver by in vivo adenovirus-mediated gene transfer of the antiapoptotic Bcl-2 gene. Ann Surg 1999; 230:185-92.

35. Selzner M, Sindram D, Yadav S, et al. Bcl-2 transgenic mice are resistant to ischemia/reperfusion injury of the liver: A new strategy for the prevention of hepatocellular injury. Hepatology 1999; 30:386A. 
36. Calabrese F, Pontisso P, Pettenazzo E, et al. Liver cell apoptosis in chronic hepatitis $\mathrm{C}$ correlates with histological but not biochemical activity or serum HCV-RNA levels. Hepatology 2000; 31:1153-1159.

37. Krams S, Egawa H, Quinn M, et al. Apoptosis as a mechanism of cell death in liver allograft rejection. Transplantation 1995; 59:621-625.

38. Peralta C, Closa D, Xaus C, et al. Hepatic preconditioning in rats is defined by a balance of adenosine and xanthine. Hepatology 1998; 28:768-773.

39. Yamaguchi T, Terakado M, Horio F, et al. Role of bilirubin as an antioxidant in an ischemia-reperfusion of rat liver and induction of heme oxygenase. Biochem Biophys Res Commun 1996; 223:129-135.

40. Zwacka RM, Zhang Y, Zhou W, et al. Ischemia/reperfusion injury in the liver of BALB/c mice activates AP-1 and nuclear factor kappa-B independently of I-kappa-B degradation. Hepatology 1998; 28:1022-1030.

41. Tacchini L, Radice L, Pogliaghi G, et al. Differential activation of heat shock and nuclear factor kappa-B transcription factors in postischemic reperfused rat liver. Hepatology 1997; 26:186-191.

42. Sindram D, Porte RJ, Hoffman MR, et al. Platelets induce sinusoidal endothelial cell apoptosis upon reperfusion of the cold ischemic rat liver. Gastroenterology 2000; 118:183-191.
43. Cutrin JC, Boveris A, Zingaro B, et al. In situ determination by surface chemiluminescence of temporal relationships between evolving warm ischemia-reperfusion injury in rat liver and phagocyte activation and recruitment. Hepatology 2000; 31:622-632.

44. Lentsch AB, Yoshidome H, Kato A, et al. Requirement for interleukin-12 in the pathogenesis of warm hepatic ischemia/reperfusion injury in mice. Hepatology 1999; 30:1448-1453.

45. Belghiti J, Hiramatsu K, Benoist S, et al. Seven hundred forty-seven hepatectomies in the 1990s: An update to evaluate the actual risk of liver resection. J Am Coll Surg 2000; 191:38-46.

46. Selzner M, Rudiger H, Sindram D, et al. Ischemia/reperfusion injury in the steatotic liver. Gastroenterology 2000; 118:A1002.

47. Fong Y, Sun RL, Jarnagin W, et al. An analysis of 412 cases of hepatocellular carcinoma at a Western center. Ann Surg 1999; 229: $790-800$.

48. Eleftheriadis E, Kotzampassi K, Iliadis S, et al. Hepatic tissue microcirculation, oxygenation and energy charge in ischemia-reperfusion subjected cirrhotic rat liver. Hepato-Gastroenterology 1997; 44:11871192. 